\title{
Efficacy and safety assessment of apatinib in patients with advanced gastric cancer: a meta-analysis
}

This article was published in the following Dove Press journal: OncoTargets and Therapy

\author{
Jianxin Chen' \\ Junhui Wang ${ }^{2}$ \\ 'Department of Medical Oncology, \\ Quzhou People's Hospital, Quzhou, \\ 324000 , Zhejiang, People's Republic \\ of China; ${ }^{2}$ Department of Radiation \\ Oncology, Quzhou People's Hospital, \\ Quzhou, 324000, Zhejiang, People's \\ Republic of China
}

Aim: This meta-analysis was performed to evaluate the efficacy and safety of apatinib in patients with advanced gastric cancer (AGC).

Methods: After evaluating the inclusion and exclusion criteria, the data of eligible randomized clinical trials (RCTs) were extracted. Outcomes including objective response rate (ORR), disease control rate (DCR), and adverse events (AEs) were analyzed in the meta-analysis.

Results: Data of 1,069 patients from 13 RCTs were statistically analyzed. Pooled odds ratio (OR) for ORR and DCR was found to be 0.46 (95\% confidence interval [CI]: 0.33, 0.64; $P<0.00001)$ and 0.23 (95\% CI: 0.15, 0.36; $P<0.00001)$, respectively. Compared with placebo, apatinib showed statistical significance in AEs at any grade, including leucopenia, neutropenia, thrombocytopenia, diarrhea, hypertension, proteinuria, hand-foot syndrome, and fatigue (all $P<0.05)$.

Conclusion: The results of our meta-analysis revealed that apatinib shows short-term efficacy over no-apatinib regimens or placebo regardless of its use as first- or second-line chemotherapy or for further treatment in patients with AGC accompanied with apparent AEs of any grade.

Keywords: objective response rate, disease control rate, adverse events

\section{Introduction}

Gastric cancer is one of the most common cancers worldwide with approximately 1 million new cases and more than 700,000 deaths reported globally each year. ${ }^{1}$ Although the treatment of gastric cancer has been improved significantly with the development of surgery, chemotherapy, and radiotherapy, the overall survival (OS) of patients with advanced gastric cancer (AGC) or metastatic cancer still remains less than 1 year. ${ }^{2}$ Fortunately, valuation of targeted therapy has been confirmed in recent years. Trastuzumab has demonstrated to extend the median OS of patients with human epidermal growth factor receptor-2-positive AGC or cancer of the gastroesophageal junction by 2.7 months (hazard ratio $[\mathrm{HR}]=0.74,95 \%$ confidence interval (CI): 0.60 , $0.91 ; P=0.0046){ }^{3}$ In terms of antiangiogenesis, bevacizumab demonstrated efficacy in AGC with a prolonged progression-free survival (PFS) compared to placebo (6.7 versus 5.3 months, $\mathrm{HR}=0.80,95 \% \mathrm{CI}: 0.68-0.93 ; P=0.0037) .{ }^{4}$ Another randomized, placebo-controlled, Phase III clinical trial on ramucirumab revealed that patients with pretreated AGC would benefit from ramucirumab with a prolonged PFS as well as $\mathrm{OS} ;{ }^{5}$ however, it causes significant adverse events (AEs) such as proteinuria and hypertension. ${ }^{5}$ Therefore, there is an urgent need to develop chemotherapeutic agents with higher efficacy and lower toxicities that can be used in the treatment of AGC. $\mathrm{BY}$
hereby accept the Terms. Non-commercial uses of the work are permitted without any further permission from Dove Medical Press Limited, provided the work is properly attributed. For permission for commercial use of this work, please see paragraphs 4.2 and 5 of our Terms (https://www.dovepress.com/terms.php). 
Apatinib, a small-molecule tyrosine kinase inhibitor, selectively binds to and strongly inhibits VEGFR-2, thereby decreasing VEGF-mediated endothelial cell migration, proliferation, and density of the tumor microvasculature. A randomized, double-blind, placebo-controlled Phase III trial demonstrated that apatinib treatment significantly improved OS (6.5 versus 4.7 months, HR $=0.709,95 \%$ CI: $0.537,0.937$; $P=0.0156)$ and $\mathrm{PFS}$ ( 2.6 versus 1.8 months, HR $=0.444,95 \%$ CI: $0.331,0.595 ; P<0.001)$ with an acceptable safety profile in patients with AGC refractory to two or more lines of prior chemotherapy. ${ }^{6}$ In addition, numerous randomized clinical trials (RCTs) had been developed to evaluate the efficacy and safety in patients with AGC, especially in People's Republic of China in recent years. However, according to relative literatures (Table 1), the efficacy and safety assessment of apatinib for treatment in patients with AGC were presented with inconsistency.

Therefore, in this review, we aimed to provide a detailed evaluation of the efficacy and safety profile of apatinib as the first- and second-line chemotherapeutic agent or for further treatment in patients with AGC.

\section{Materials and methods}

\section{Search strategy}

The following databases were searched for the publications in English and Chinese: PubMed, Google Scholar, Cochrane Library, EBSCO Publishing, ClinicalKey, Ovid, and China National Knowledge Infrastructure (CNKI). Meeting abstracts including American Society of Clinical Oncology (ASCO) and European Society of Medical Oncology (ESMO) were also reviewed. The date of the last search was July 4, 2017. The definite words applied for searching in English were "apatinib" and "gastric cancer" or "stomach cancer" in the titles, whereas the definite words for searching in Chinese (for CNKI) were "apatinib" or "Ai tan" and "Wei ai" included in the titles.

\section{Selection criteria} Inclusion criteria

1. RCTs including patients aged above 18 years with Eastern Cooperative Oncology Group (ECOG) performance status of 0,1 , or 2 and having at least one measurable lesion as defined by RECIST version $1.1{ }^{7}$

Table I The characteristics of included studies

\begin{tabular}{|c|c|c|c|c|}
\hline References & $\begin{array}{l}\text { Status of } \\
\text { treatment }\end{array}$ & Treatment arms & Cases & End points \\
\hline \multirow[t]{2}{*}{ Li et al ${ }^{6}$} & Second-line & Apatinib & 181 & ORR/DCR/PFS/OS \\
\hline & or further & Placebo & 92 & \\
\hline \multirow[t]{3}{*}{$\overline{\mathrm{Li} \text { et } \mathrm{al}} \mathrm{I}^{10}$} & Second-line & Placebo & 48 & ORR/DCR/PFS/OS \\
\hline & or further & Apatinib 850 mg Qd* & 48 & \\
\hline & & Apatinib 425 mg Bid** & 48 & \\
\hline \multirow{2}{*}{$\overline{Z h u ~ e t ~ a l " l ~}$} & Second-line & PTX or 5 -FU or CPT-II & 39 & ORR/DCR \\
\hline & or further & Above+apatinib & 32 & \\
\hline \multirow[t]{2}{*}{$\overline{\text { Gao et } \mathrm{al}^{12}}$} & Second-line & Apatinib+S-I & 16 & ORR/DCR \\
\hline & or further & S-I & 15 & \\
\hline \multirow[t]{2}{*}{ Gao and Fang ${ }^{13}$} & First-line & Apatinib+FOLFIRI & 40 & ORR/DCR \\
\hline & & FOLFIRI*** & 40 & \\
\hline \multirow[t]{2}{*}{$\overline{\text { Wen et al }}{ }^{14}$} & First-line & Apatinib+SOX & 45 & ORR/DCR \\
\hline & & SOX**** & 45 & \\
\hline \multirow[t]{2}{*}{ Chen et al ${ }^{15}$} & Second-line & Apatinib & 50 & ORR \\
\hline & or further & S-I & 49 & \\
\hline \multirow[t]{2}{*}{ Ding et $\mathrm{al}^{16}$} & Second-line & Apatinib & 30 & ORR/DCR \\
\hline & or further & S-I & 30 & \\
\hline \multirow[t]{2}{*}{ Lang et $\mathrm{al}^{17}$} & Second-line & Apatinib & 14 & ORR/DCR \\
\hline & or further & Placebo & 14 & \\
\hline \multirow[t]{2}{*}{$\overline{\text { Fan et } \mathrm{al}^{18}}$} & First-line & Apatinib+S-I & 15 & ORR/DCR \\
\hline & & S-I & 15 & \\
\hline \multirow[t]{2}{*}{ Wang et a ${ }^{19}$} & First-line & Apatinib+PS & 29 & ORR \\
\hline & & PS****** & 29 & \\
\hline \multirow[t]{2}{*}{ Wang et al ${ }^{20}$} & Second-line & Apatinib & 25 & ORR \\
\hline & or further & S-I & 25 & \\
\hline \multirow[t]{2}{*}{ Xue and $\mathrm{Cui}^{21}$} & Second-line & Apatinib & 25 & ORR \\
\hline & or further & Placebo & 30 & \\
\hline
\end{tabular}

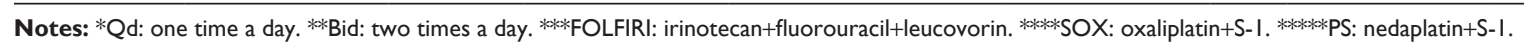

Abbreviations: 5-FU, 5-fluorouracil; CPT-II, irinotecan; DCR, disease control rate; ORR, objective response rate; OS, overall survival; PFS, progression-free survival; PTX, paclitaxel; S-I, tegafur gimeracil oteracil potassium capsule. 
2. Patients included in the original research should be histologically or cytologically diagnosed as having advanced (IIIB/IIIC/IV) GC, cancer staging performed according to the 7 th edition of the American Joint Committee on Cancer (AJCC). ${ }^{8}$

3. Prospective Phase II/III RCTs that evaluated apatinib alone or apatinib-based regimens as the first- or second-line chemotherapy or used for further treatment in patients with AGC. Patients who received apatinib therapy as the second-line chemotherapy or for further treatment should have experienced failure of standard regimens such as 5-fluorouracil and/or platinum-based regimens. Treatment failure was defined as intolerable AEs or disease progression during treatment with chemotherapy.

4. The patients included should be randomized into two arms: one managed with apatinib and the other arm handled with standard chemotherapy regimens or placebo for the control.

5. One of the following results should be reported: objective response rate (ORR), disease control rate (DCR), or AEs.

\section{Exclusion criteria}

1. Non-RCTs, meta-analysis, reviews, case reports, and correspondings were excluded.

2. Not prospective Phase II/III RCTs.

3. Studies without the field of interest of this review.

4. If the language was not English or Chinese.

\section{Data extraction}

1. Basic characteristics of RCTs including first author's names, year of publication, status of treatment, treatment regimens, patients' indications, and end points.

2. End points in this meta-analysis were ORR (including rate of complete response plus partial response), DCR (including rate of complete response plus partial response plus stable disease), and any grade AEs including hematological toxicities, digestive events, and general events; ORR accounts for the primary end point. AEs were assessed with National Cancer Institute Common Terminology Criteria for Adverse Events version 4.0. ${ }^{9}$

3. The meta-analysis was executed with the guidelines by Preferred Reporting Items for Systematic Reviews and Meta-Analyses (PRISMA) report.

\section{Statistical analysis}

The data were analyzed with RevMan 5.3 software. For efficacy and safety evaluation, ORR, DCR, and AEs were calculated with odds ratio (OR). A $P$-value $<0.05$ was considered statistically significant. Cochrane $Q$ test and inconsistency statistic $\left(I^{2}\right)$ were used to evaluate the heterogeneity among the included RCTs. Fixed-effect model was adopted for the existence of heterogeneity, in which $P>0.1$ and $I^{2}<50 \%$ and developed with fixed-effect model, whereas in case of $P<0.1$ and $I^{2}>50 \%$, random-effect model was adopted. Publication bias was assessed using funnel plots with primary end point.

\section{Results \\ Search results}

According to PRISMA diagram shown in Figure 1, 502 RCTs were obtained from PubMed $(n=210)$ and other databases ( $n=292)$. After deleting the duplicates, 413 RCTs were left, of which 86 RCTs were selected by reading titles. With the limitation to RCTs of apatinib in gastric cancer, 13 studies were included for the final analysis. All included RCTs used apatinib as the first- or second-line chemotherapy or for further treatment in patients with AGC. Table 1 shows treatment regimens and group control settings. The studies included did not report any relationship between age, sex, and clinical outcomes $(P>0.05)$.

A total of 1,069 patients were included in the analysis, all of whom received apatinib alone or apatinib-based regimens as treatment.

\section{Efficacy results}

The pooled OR for ORR was found to be 0.46 (95\% CI: $0.33,0.64 ; P<0.00001)$. Results of ORR with the evaluation of heterogeneity between subgroups with $I^{2}$ test showed no statistically significant heterogeneity $\left(I^{2}=0 \%, P=0.77\right)$ (Figure 2A).

Results of DCR with the evaluation of heterogeneity between subgroups with $I^{2}$ test showed statistically significant heterogeneity $\left(I^{2}=76.5 \%, P=0.04\right)$ (Figure 2B). After switching to Mantel-Haenzel $(\mathrm{M}-\mathrm{H})$ random-effect model for analysis, the pooled OR for DCR was found to be 0.23 (95\% CI: $0.15,0.36 ; P<0.00001)$.

\section{AEs}

For analysis of hematological toxicities with $\mathrm{M}-\mathrm{H}$ random model, the pooled OR for leukopenia of any grade was found to be 5.73 (95\% CI: 2.90, 11.32; $P<0.00001)$. The pooled ORs for neutropenia, anemia, and thrombocytopenia of any grade were found to be 3.38 (95\% CI: $1.57,7.29 ; P=0.002)$, 1.25 (95\% CI: $0.83,1.88 ; P=0.28)$, and 2.25 (95\% CI: 1.30 , 3.90; $P=0.004)$, respectively (Figure $3 \mathrm{~A}$ ). 


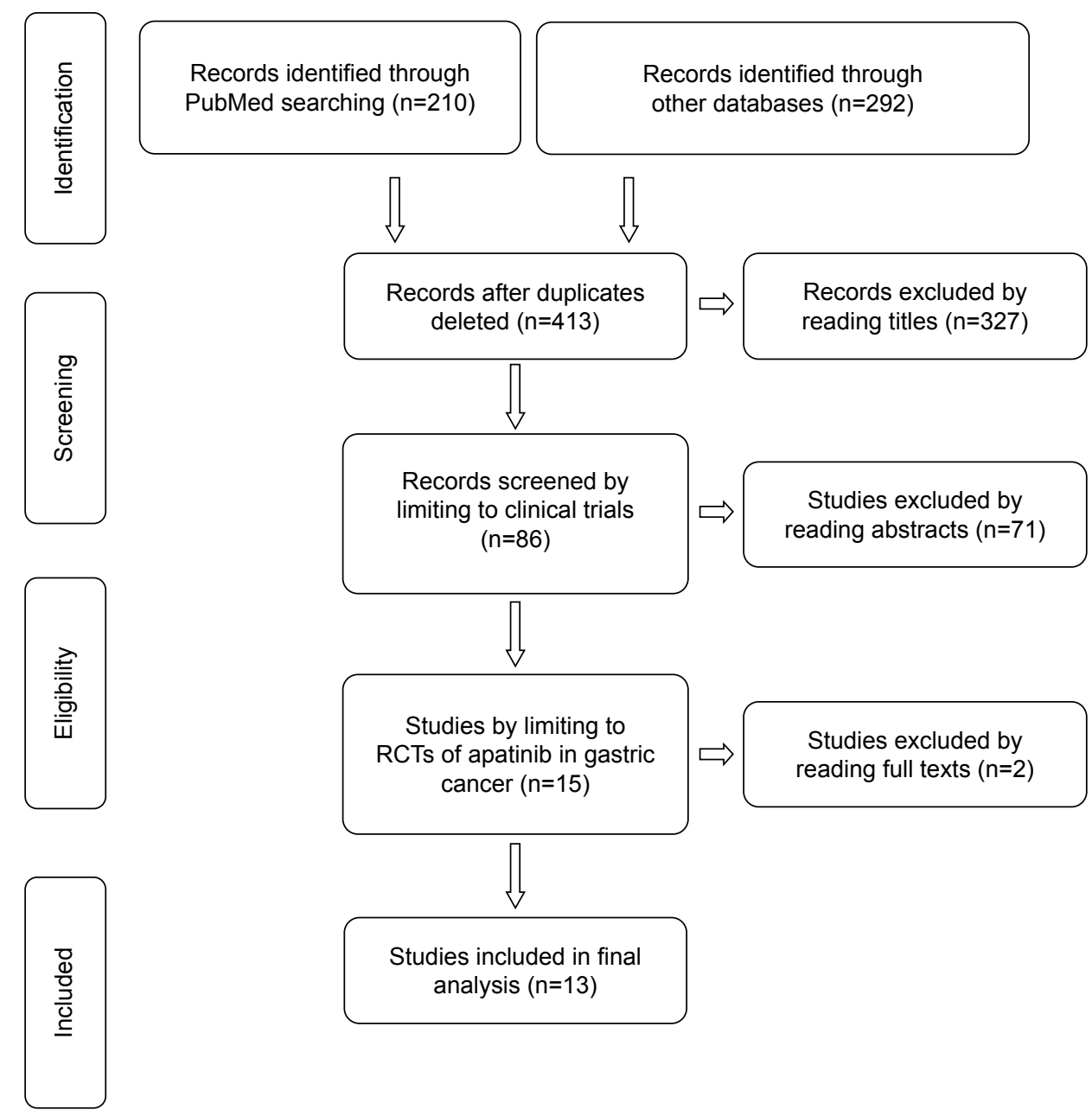

Figure I Study selection procedure with PRISMA flow diagram.

Abbreviations: PRISMA, Preferred Reporting Items for Systematic Reviews and Meta-Analyses; RCTs, randomized controlled trials.

In terms of digestive events, the pooled ORs for diarrhea and nausea/vomiting of any grade were found to be 2.88 (95\% CI: 1.46, 5.68; $P=0.002$ ) and 1.24 (95\% CI: 0.74, 2.08; $P=0.42$ ), respectively (Figure 3B).

When referring to the general events, the pooled ORs for hypertension, proteinuria, hand-foot syndrome, and fatigue of any grade were found to be 10.76 (95\% CI: 5.94, 17.49; $P<0.00001), 4.55$ (95\% CI: 2.78, 7.44; $P<0.00001), 5.15$ (95\% CI: 2.91, 9.11; $P<0.00001$ ), and 1.67 (95\% CI: 1.01 , 2.76; $P=0.04$ ), respectively (Figure $3 \mathrm{C} 1$ and 2 ).

\section{Publication bias}

Funnel plot with ORR did not reveal any significant publication bias (Figure 4).

\section{Discussion}

To our knowledge, this is the first meta-analysis to evaluate the efficacy and safety of apatinib in patients with AGC. The results of this meta-analysis have demonstrated that apatinib shows short-term efficacy (ORR: 0.46, 95\% CI: 0.33, 0.64; $P<0.00001$; DCR: $0.23,95 \%$ CI: $0.15,0.36 ; P<0.00001$ ) over no-apatinib regimens or placebo regardless of apatinib being administered as first- or second-line chemotherapy or for further treatment in patients with AGC. In addition, apatinib has been accompanied with substantial AEs of any grade, including hematological toxicities, digestive events, and general events, such as hypertension, proteinuria, hand-foot syndrome, and fatigue compared to the control treatment.

Apatinib, was invented in People's Republic of China and approved by the China Food and Drug Administration (CFDA) for further treatment in patients with AGC or esophagogastric junction adenocarcinoma on October 17, 2014. ${ }^{22}$ This is the reason why majority of the relative clinical trials have been developed in the past 1 or 2 years in People's Republic of China. During our analysis of ORR, all included studies were pooled to gather statistics, three of which had been designed as comparative trials between apatinib and tegafur gimeracil oteracil potassium capsule (S-1) which had already 


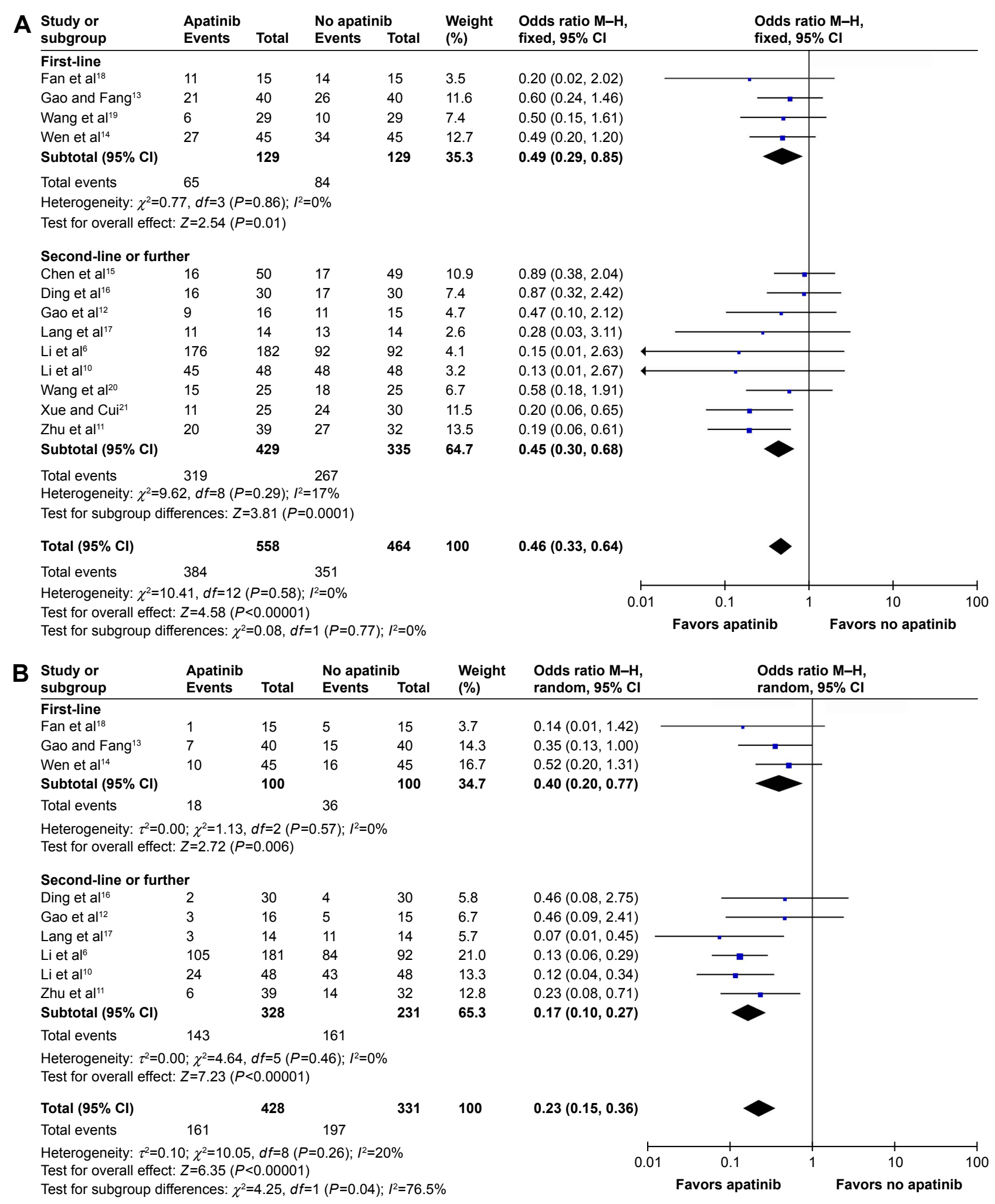

Figure 2 Forest plot of ORR (A) and DCR (B) between apatinib-alone or apatinib-based regimens and no-apatinib groups. Abbreviations: $\mathrm{Cl}$, confidence interval; DCR, disease control rate; $\mathrm{M}-\mathrm{H}$, Mantel-Haenzel; ORR, objective response rate.

been recommended for standard chemotherapy in People's Republic of China according to JCOG9912. ${ }^{23}$ Owing to the second-line chemotherapeutic status of apatinib or S-1 in the three trials, they were considered for the final analysis..$^{15,16,20}$
With the pooled analysis of short-term efficacy including ORR and DCR, it is evident that apatinib can shrink the size of the tumor with or without chemotherapy; its efficacy was found to be in accordance with another antiangiogenic agent, 
ramucirumab. According to some Phase III studies, a significant ORR was observed in treatment group with ramucirumab compared to placebo group, including in patients with AGC or gastroesophageal junction adenocarcinoma, ${ }^{5,24}$ non-small-cell lung cancer (NSCLC), ${ }^{25}$ and advanced hepatocellular carcinoma. ${ }^{26}$ Meanwhile, ramucirumab has shown the benefits of improved OS in patients with solid carcinomas such as gastric cancer, NSCLC, and colorectal cancer. ${ }^{27}$ However,

A

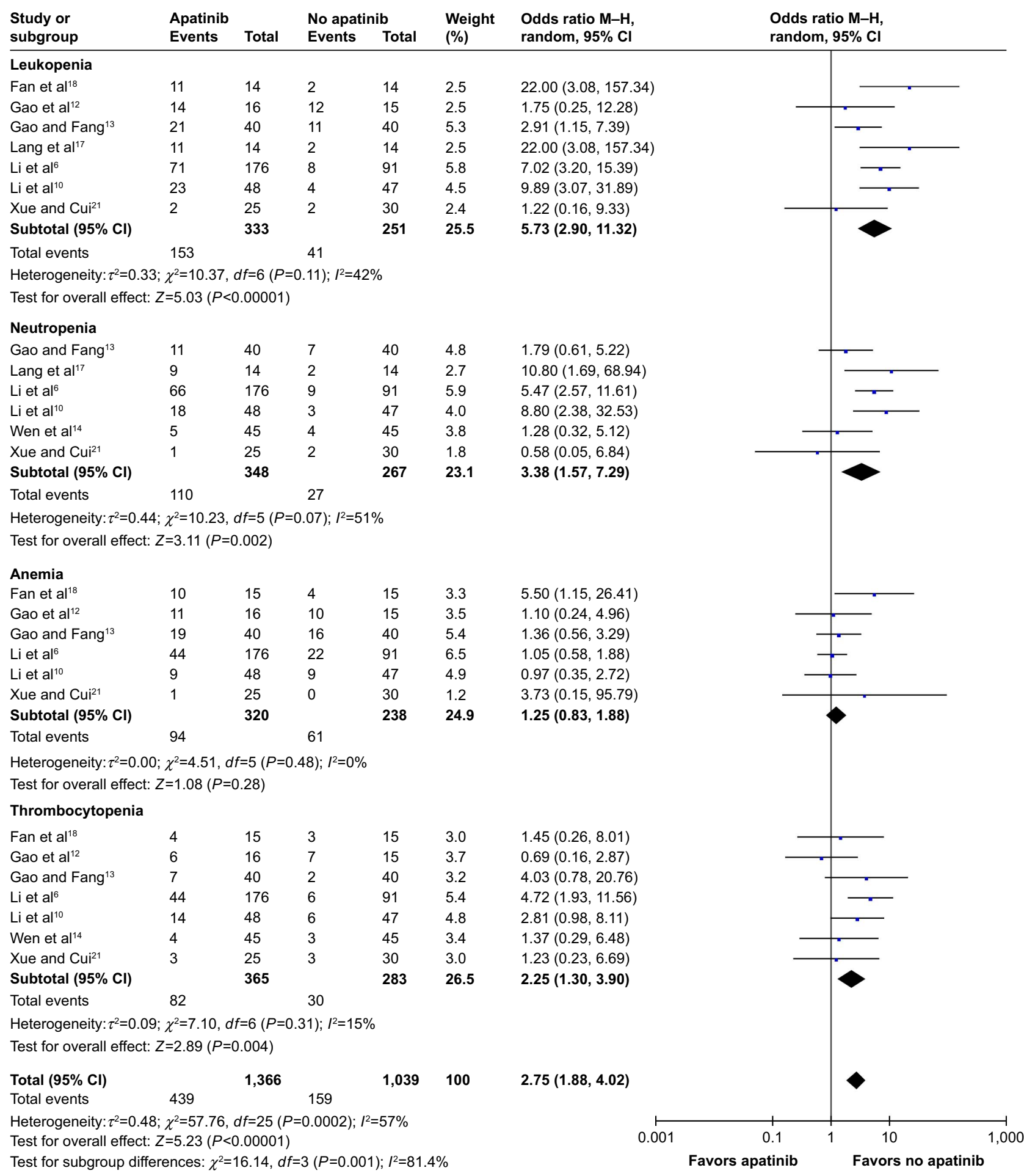

Figure 3 (Continued) 
C2

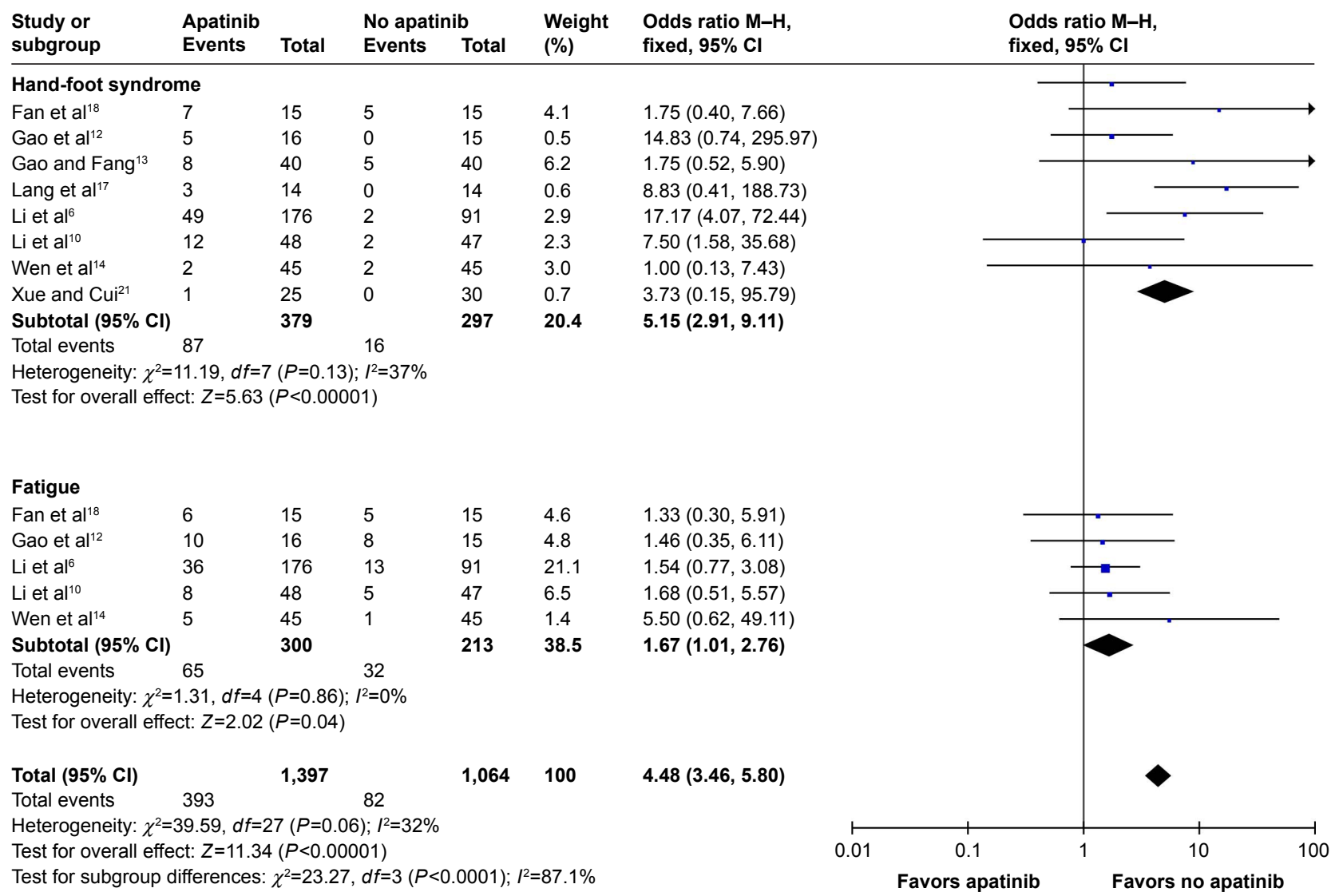

Figure 3 Forest plot of hematological toxicity (A), digestive events $(\mathbf{B})$, and general events $(\mathbf{C l}$ and $\mathbf{C} 2)$ between apatinib-alone or apatinib-based regimens and noapatinib groups.

Abbreviations: $\mathrm{Cl}$, confidence interval; $\mathrm{M}-\mathrm{H}$, Mantel-Haenzel.

long-term efficacy of apatinib in improving PFS or OS has not been evaluated in this analysis due to the data deficiencies. Outcomes such as PFS and OS have been reported for apatinib in only two RCTs, ${ }^{6,10}$ which might be due to the fact that the

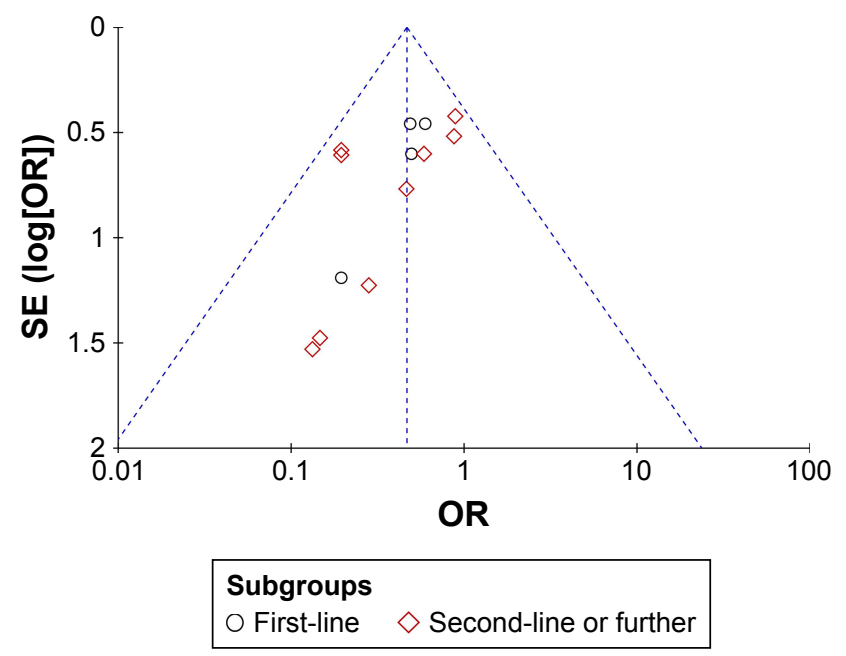

Figure 4 Funnel plot for publication bias with ORR.

Abbreviations: OR, odds ratio; ORR, objective response rate; SE, standard error. majority of studies were conducted in years 2016 and 2017 with the immature outcomes of PFS and OS. According to the aforementioned two RCTs, apatinib shows long-term efficacy based on outcomes such as PFS and OS, which might indicate its promising role as an anticancer drug in the future.

Although majority of the trials report that the AEs of apatinib are tolerable, ${ }^{6,13,19}$ the pooled analysis outcomes of AEs, especially AEs of any grade, including hematological toxicities, digestive events, and general events, showed a significant difference (Figure 3). During the final analysis of AEs, the full-analysis set population was used instead of intent-to-treat population. In addition, three RCTs, which had been designed as the comparison of apatinib and S-1, had been excluded in the final analysis of AEs due to the probable bias caused by S-1 instead of placebo or other balanced regimens between apatinib groups and the control ones. ${ }^{15,16,20}$ As shown in Figure 3, AEs of any grade, including leucopenia, neutropenia, thrombocytopenia, diarrhea, hypertension, proteinuria, hand-foot syndrome, and fatigue had been deemed as statistically significant $(P<0.05)$. This might 
have restricted the application of apatinib in patients with AGC, especially with poor performance status of 2 or 3 . Ramucirumab has been reported to show AEs, including proteinuria, ${ }^{28}$ stomatitis and gastrointestinal perforation, ${ }^{29}$ hypertension, ${ }^{30}$ and hematological toxicity. ${ }^{31}$ However, RCTs included in this meta-analysis did not report gastrointestinal perforation during the treatment with apatinib. The safety comparison between ramucirumab and apatinib is still controversial, which needs further and more direct assessment.

Other than AGC, the efficacy and safety of apatinib has been explored in more cancers, including NSCLC, osteosarcoma, advanced soft tissue sarcoma, metastatic breast cancer, prostate cancer, hepatocellular carcinoma, and colorectal cancer according to ASCO annual meeting 2017 (meeting. ascopubs.org). We believe that more promising results with regard to apatinib will be presented in the near future.

This meta-analysis has some limitations. First, all the RCTs included in the analysis were performed on Asian population (Chinese), because of the regional employment of apatinib in recent years. Missing data in Caucasian population might lead to a racial bias in the final analysis. Second, except for the two RCTs, ${ }^{6,10}$ the deficiency of long-term follow-up period makes us confused with the survival benefit of apatinib, which is supposed to be the paramount norm in the treatment in advanced cancers. Confirmatory prospective clinical studies in this regard are highly warranted.

\section{Conclusion}

This meta-analysis has revealed that apatinib has an advantage of short-term efficacy over no-apatinib regimens or placebo regardless of its use as first- or second-line chemotherapeutic agent or for further treatment in patients with AGC accompanied with apparent AEs of any grade.

\section{Disclosure}

The authors report no conflicts of interest in this work.

\section{References}

1. Jemal A, Bray F, Center MM, Ferlay J, Ward E, Forman D. Global cancer statistics. CA Cancer J Clin. 2011;61(2):69-90.

2. Cunningham D, Allum WH, Stenning SP, et al; MAGIC Trial Participants. Perioperative chemotherapy versus surgery alone for resectable gastroesophageal cancer. $N$ Engl J Med. 2006;355(1):11-20.

3. Bang YJ, Van Cutsem E, Feyereislova A, et al; ToGA Trial Investigators. Trastuzumab in combination with chemotherapy versus chemotherapy alone for treatment of HER2-positive advanced gastric or gastrooesophageal junction cancer (ToGA): a phase 3 , open-label, randomised controlled trial. Lancet. 2010;376(9742):687-697.

4. Ohtsu A, Shah MA, Van Cutsem E, et al. Bevacizumab in combination with chemotherapy as first-line therapy in advanced gastric cancer: a randomized, double-blind, placebo-controlled phase III study. J Clin Oncol. 2011;29(30):3968-3976.
5. Fuchs CS, Tomasek J, Yong CJ, et al; REGARD Trial Investigators. Ramucirumab monotherapy for previously treated advanced gastric or gastro-oesophageal junction adenocarcinoma (REGARD): an international, randomised, multicentre, placebo-controlled, phase 3 trial. Lancet. 2014;383(9911):31-39.

6. Li J, Qin S, Xu J, et al. Randomized, double-blind, placebo-controlled phase III trial of apatinib in patients with chemotherapy-refractory advanced or metastatic adenocarcinoma of the stomach or gastroesophageal junction. J Clin Oncol. 2016;34(13):1448-1454.

7. Eisenhauer EA, Therasse P, Bogaerts J, et al. New response evaluation criteria in solid tumours: revised RECIST guideline (version 1.1). Eur J Cancer. 2009;45(2):228-247.

8. Edge SB, Compton CC. The American Joint Committee on Cancer: the 7th edition of the AJCC cancer staging manual and the future of TNM. Ann Surg Oncol. 2010;17(6):1471-1474.

9. Chen AP, Setser A, Anadkat MJ, et al. Grading dermatologic adverse events of cancer treatments: the Common Terminology Criteria for Adverse Events Version 4.0. J Am Acad Dermatol. 2012;67(5): $1025-1039$.

10. Li J, Qin S, Xu J, et al. Apatinib for chemotherapy-refractory advanced metastatic gastric cancer: results from a randomized, placebocontrolled, parallel-arm, phase II trial. J Clin Oncol. 2013;31(26): 3219-3225.

11. Zhu H, Sun X, Zhou Q, Chen L, Chen J, Wu P. Clinical study of Apatinib combined chemotherapy in the treatment of advanced gastric cancer. Chin J Surg Oncol. 2016;8(6):394-396.

12. Gao J, Han T, Piao Y, et al. Apatinib combined tegafur in treatment for elderly or emaciated patients with advanced gastric cancer. Clin J Med Offic. 2017;45(1):9-12.

13. Gao F, Fang X. The short term efficacy of apatinib combined with CPT-11 and 5-FU in patients with advanced gastric cancer. Clin Album. 2016;12(6):624-625.

14. Wen F, Xiang Y, Wang L. Clinical trial of apatinib tablets in the treatment of advanced gastric cancer. Clin Study. 2017;33(7):589-591.

15. Chen Z, Zheng Q, Guo B. Comparison of apatinib and S-1 in second line treatment of advanced gastric cancer. Chin J Clin Ratl Drug Use. 2017;10(6A):79-80.

16. Ding L, Xia Y, Wang W, Meng M, Yao R, Bi J. Efficacy and prognosis assessment of apatinib and S-1 in patients with advanced gastric cancer. J Anhui Tech Coll Health. 2017;16(2):48-49.

17. Lang F, Zhao Y, Fan P. Clinical efficacy and safety of apatinib mesylatefor patients with advanced gastric cancer. Pract J Cancer. 2017;32(6): 996-998.

18. Fan X, Lv H, Chen B, et al. Clinical study of Apatinib combined with S-1 in the treatment of patients with advanced gastric cancer and review of literature. Chin J Med Front. 2017;9(2):63-67.

19. Wang D, Song S, Wang K. Efficacy assessment of apatinib combined with chemotherapy in patients with advanced gastric cancer. World Latest Med Inf. 2016;16(87):130-131.

20. Wang P, Shang N, Liu Z, Dong X. Clinical research of apatinib combined with S-1 in patients with advanced gastric cancer. J Taishan Med Coll. 2016;37(8):919-920.

21. Xue D, Cui L. Efficacy assessment of apatinib in the treatment after failure of second line chemotherapy in patients with advanced gastric cancer. Contemp Med Symp. 2016;14(22):126-127.

22. Qin S, Li J. Chinese specialist consensus on the treatment of gastric cancer with apatinib. Chin Clin Oncol. 2015;20(9):841-847.

23. Takashima A, Boku N, Kato K, et al. Survival prolongation after treatment failure of first-line chemotherapy in patients with advanced gastric cancer: combined analysis of the Japan Clinical Oncology group trials JCOG9205 and JCOG9912. Gastric Cancer. 2014;17(3): $522-528$.

24. Wilke H, Muro K, Van Cutsem E, et al; RAINBOW Study Group. Ramucirumab plus paclitaxel versus placebo plus paclitaxel in patients with previously treated advanced gastric or gastro-oesophageal junction adenocarcinoma (RAINBOW): a double-blind, randomised phase 3 trial. Lancet Oncol. 2014;15(11):1224-1235. 
25. Garon EB, Ciuleanu TE, Arrieta O, et al. Ramucirumab plus docetaxel versus placebo plus docetaxel for second-line treatment of stage IV non-small-cell lung cancer after disease progression on platinum-based therapy (REVEL): a multicentre, double-blind, randomised phase 3 trial. Lancet. 2014;384(9944):665-673.

26. Zhu AX, Park JO, Ryoo BY, et al. Ramucirumab versus placebo as second-line treatment in patients with advanced hepatocellular carcinoma following first-line therapy with sorafenib (REACH): a randomised, double-blind, multicentre, phase 3 trial. Lancet Oncol. 2015; 16(7):859-870.

27. Tabernero J, Yoshino T, Cohn AL, et al; RAISE Study Investigators. Ramucirumab versus placebo in combination with second-line FOLFIRI in patients with metastatic colorectal carcinoma that progressed during or after first-line therapy with bevacizumab, oxaliplatin, and a fluoropyrimidine (RAISE): a randomised, double-blind, multicentre, phase 3 study. Lancet Oncol. 2015;16(5):499-508.
28. Abdel-Rahman O, ElHalawani H. Proteinuria in patients with solid tumors treated with ramucirumab: a systematic review and metaanalysis. Chemotherapy. 2014;60(5-6):325-333.

29. Abdel-Rahman O, ElHalawani H. Risk of oral and gastrointestinal mucosal injury in patients with solid tumors treated with ramucirumab: a systematic review and meta-analysis. Expert Opin Drug Saf. 2015; 14(10):1495-1506.

30. Abdel-Rahman O, ElHalawani H. Risk of cardiovascular adverse events in patients with solid tumors treated with ramucirumab: a meta analysis and summary of other VEGF targeted agents. Crit Rev Oncol Hematol. 2016;102:89-100.

31. Abdel-Rahman O, ElHalawani H. Risk of hematological toxicities in patients with solid tumors treated with ramucirumab: a meta-analysis. Future Oncol. 2015;11(21):2949-2961.
OncoTargets and Therapy

\section{Publish your work in this journal}

OncoTargets and Therapy is an international, peer-reviewed, open access journal focusing on the pathological basis of all cancers, potential targets for therapy and treatment protocols employed to improve the management of cancer patients. The journal also focuses on the impact of management programs and new therapeutic agents and protocols on

\section{Dovepress}

patient perspectives such as quality of life, adherence and satisfaction. The manuscript management system is completely online and includes a very quick and fair peer-review system, which is all easy to use. Visit http://www.dovepress.com/testimonials.php to read real quotes from published authors. 